\title{
Near-Field Localization of Single Au Cubes: A Group Theory Description
}

\author{
Sarra Mitiche, ${ }^{\dagger}$ Sylvie Marguet, ${ }^{\dagger}$ Fabrice Charra, ${ }^{\dagger}{ }^{\dagger}$ and Ludovic Douillard ${ }^{*}{ }^{\dagger} \odot$ \\ ${ }^{\dagger}$ SPEC, CEA, CNRS and ${ }^{\ddagger}$ NIMBE, CEA, CNRS, Université Paris-Saclay, CEA Saclay, F-91191 Gif sur Yvette, France
}

Supporting Information

ABSTRACT: A simple group theory approach is proposed to predict the charge distribution of low order localized surface plasmon resonances (LSPRs) of finite metallic particles of basic geometries. As an illustration, the case of randomly oriented Au colloidal particles of cubic $C_{4 v}$ symmetry excited at dipolar resonance is presented. The symmetry approach is confirmed by numerical simulations carried out by the boundary element method. Experimental validation is achieved by high-resolution subwavelength near-field mapping conducted by photoemission electron microscopy.

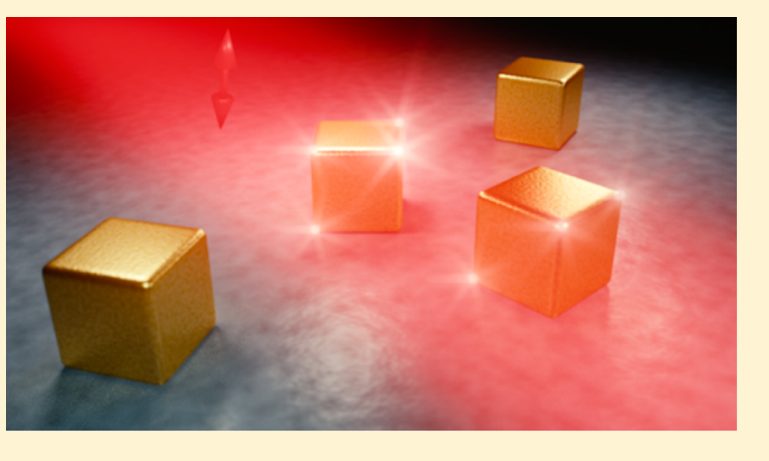

\section{INTRODUCTION}

Light-matter interaction at the ultimate scale can exhibit a physical behavior at variance with what is commonly encountered at the macroscopic scale. Localized surface plasmon resonances (LSPRs), collective oscillations of the conduction electrons at a metallic/dielectric interface, are behind the remarkable optical properties of nanosized metallic particles. ${ }^{1}$ Plasmonics, a major part of nanophotonics, holds great promise for a broad range of application domains including artificial photosynthesis, ${ }^{2,3}$ biosensing, ${ }^{4-6}$ cancer therapy, energy harvesting, ${ }^{8}$ imaging, ${ }^{9}$ plasmon laser, ${ }^{10}$ and surface-enhanced spectrometries. ${ }^{11}$ By contrast, only a few applications have appeared on the market, dealing mainly with sensing applications. ${ }^{12}$ Among the milestone steps likely to focus on technological interests, the precise manipulation of light at the nanometer scale is certainly at the top of the list. So far, two main routes have been cleared for the precise light manipulation at the scale of a nanostructure, namely, the coherent control and the polarization addressing of plasmonic eigenstates.

Coherent control constitutes one method of choice for subwavelength light manipulation. Relying on an interference phenomenon, it makes use of two main mechanisms: the manipulation of the near-field temporal evolution via the spectral phase shaping of a linearly polarized incident light ${ }^{13}$ and the constructive/destructive interference of near-field modes excited by a set of two orthogonally cross-polarized light components. ${ }^{14}$ The first mechanism implies an adaptive polarization pulse shaping, a complex many-parameter process, whereas the second one, more flexible, employs an open loop control scheme based on the relative phase of the two delayed incident laser pulses.

The second route exploits the selective addressing of plasmonic eigenstates of individual nanostructures of interest by specific choice of the incident light polarization. ${ }^{15-17}$ This method is simple to implement and possesses a predictive character in nature. Indeed the polarization-dependent eigenstates of an object of simple geometry (prism, cube, rectangle) can be easily analytically determined.

Following this latter approach, we present an experimental and theoretical polarization investigation of the LSPRs of individual metallic nanocubes randomly deposited on a substrate. Near-field imaging at subwavelength spatial resolution is performed using multiphoton photoemission electron microscopy (PEEM). The LSPR eigenstates are predicted within a comprehensive group theory description. Experimental and analytical results are confirmed numerically with the help of the boundary element method (BEM). ${ }^{18-20}$

This work confirms the general character of the group theory approach previously applied to the specific case of a twodimensional nano-object of trigonal symmetry. ${ }^{15}$ Here, the cubic symmetry class is considered, illustrating the physically richer case of a three-dimensional object exhibiting numerous LSPR eigenvectors, either degenerated or not. Depending on the possible resonance spectral overlaps and the polarization of the incident light, distinct final near-field distributions can be predicted.

\section{MATERIALS AND METHODS}

Sample Preparation. Regular cubes with edge sizes $\{40$, $45,75,115$, and $165 \mathrm{~nm}$ \} were prepared by colloidal chemistry in water using cetyltrimethylammonium bromide $(\mathrm{CTAB})$ as the capping agent. Cubes of edge lengths $\{40,45$, and $75 \mathrm{~nm}( \pm 3$ $\mathrm{nm})\}$ were first obtained according to an already published

Received: October 20, 2016

Revised: February 3, 2017

Published: February 6, 2017 

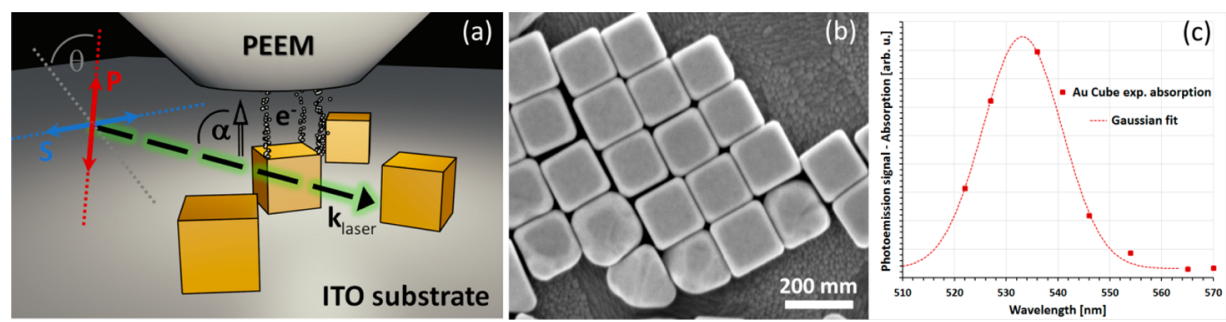

Figure 1. (a) Schematic representation of the PEEM experimental setup. (b) SEM image of Au colloidal cubes (concentrated solution). Cube edge size amounts to $165 \pm 5 \mathrm{~nm}$. (c) Average experimental light absorption of a set of 12 individual cubes. PEEM signal acquisition under p-polarization at grazing incidence.

method. ${ }^{21-23}$ Larger cubes $\{115$ and $165 \mathrm{~nm}( \pm 5 \mathrm{~nm})\}$ were obtained according to a procedure reported by Eguchi et al. ${ }^{24}$ by adding various amounts $(110 \mu \mathrm{L}$ and $60 \mu \mathrm{L})$ of the $40 \mathrm{~nm}$ cube solution, used as seed, to a growth solution $(5 \mathrm{~mL})$ containing $\mathrm{HAuCl}_{4}\left(2.0 \times 10^{-4} \mathrm{M}\right)$, ascorbic acid $\left(1.1 \times 10^{-3}\right.$ $\mathrm{M})$, and CTAB $\left(1.6 \times 10^{-2} \mathrm{M}\right)$. Small cubes are obtained in a high yield $(>90 \%)$, and larger ones are synthesized with a moderate yield $(<50 \%)$. See scanning electron microscopy (SEM) images in the Supporting Information.

Prior to photoemission investigation, colloidal objects are randomly dispersed by deposition of a liquid drop on the surface of a transparent conductive substrate. The substrate consists of a commercial polished high quality float glass coated with a $40 \mathrm{~nm}$ indium tin oxide ITO layer. ${ }^{25}$ The concentration of the solution is adapted to yield an object density of about $0.25 \mathrm{object} / \mu \mathrm{m}^{2}$. So, the corresponding averaged distance between objects amounts to $2.1 \pm 0.7 \mu \mathrm{m}$, and no significant object field coupling is expected. Figure 1 shows a typical SEM image of such a concentrated solution of gold nanoparticles.

Experiment. Photoelectron emission microscopy measurements were carried out on a previously described setup. ${ }^{26}$ In short, the photoemission process is strongly enhanced upon excitation of LSPRs, and the acquired multiphoton photoemission electron maps reflect the actual distribution of the optical near field at the surface of the metallic object under investigation. ${ }^{26-31} \mathrm{Au}$ cubes are excited under grazing incidence (angle $\alpha=72.5^{\circ}$ between $\mathrm{k}$-vector and surface normal) with the pulsed output of an optical parametric oscillator (Chameleon OPO, Coherent Inc.) pumped by a mode-locked Ti:sapphire oscillator (Chameleon Ultra II, Coherent Inc., repetition rate $80 \mathrm{MHz}$, pulse width $140 \mathrm{fs}$ ). The wavelength range in the visible is $\lambda=520-640 \mathrm{~nm}(2.38-1.94 \mathrm{eV})$. Peak power densities at the sample surface are in the range 145-300 MW/ $\mathrm{cm}^{2}$. The polarization of the laser beam can be adjusted from $\mathrm{p}-$ to s-polarization with a half-wave plate. Fundamental parameters of the illuminating beam, in particular, intensity, pulse width, and focus position, are maintained constant during the experiment, allowing point by point acquisition of wavelength- (wavelength step $5 \mathrm{~nm}$ ) and polarization-dependence (polarization step $5^{\circ}$ ) curves. The photoemission microscope (PEEM/LEEM III, Elmitec GmbH) operates under ultrahigh vacuum conditions and routinely achieves a spatial resolution down to $20 \mathrm{~nm}$ in PEEM imaging mode. In addition to PEEM imaging, the instrument can be operated in low-energy electron microscopy (LEEM) mode where the backscattered electrons are used to create an image reflecting the topography of the sample. In LEEM mode routine spatial resolution is close to $10 \mathrm{~nm}$, and topography images can be spatially correlated to the PEEM signature. For image processing LEEM and PEEM images are background corrected, and intensities are determined via signal integration over regions of interest.

Prior to polarization-dependent near-field mapping, PEEM absorption spectra (image integration) of a representative set of individual $\mathrm{Au}$ cubes of specific edge sizes are acquired to determine their resonance wavelengths. Upon increase of its edge dimension, the maximum absorption wavelength of a cube first red shifts and then goes in the reverse direction (blue shift) above a characteristic size threshold. This spectral behavior at variance with what is observed with scattering ${ }^{32-34}$ limits the number of low order LSP resonances one can excite when working with an absorption based technique. Indeed, due to the limited visible wavelength range of the OPO pulsed light source $(>520 \mathrm{~nm})$, only one full plasmon resonance is accessible. So the present investigation is limited to the cubic objects of 165 $\mathrm{nm}$ in edge size, the dipolar resonance of which (at absorption maximum) peaks at a wavelength of $533 \pm 1 \mathrm{~nm}$ (see Figure 1c).

\section{GROUP THEORY}

The plasmonic resonances of a nanoparticle can be quantitatively determined by various numerical methods, such as finite differences in time domain (FDTD), ${ }^{35-37}$ finite element $(\mathrm{FE}),^{38}$ volume integral equation (VIE),,$^{39,40}$ or surface integral equation (SIE) methods. ${ }^{18,41,42}$ These methods rely on numerical resolutions of the classical electrodynamics equations and exploit various processes of discretization allowing the use of locally appropriate analytical equations. They are efficient in handling a large variety of physical problems in nanophotonics, ${ }^{43}$ in particular the electric field distribution, and the absorption and scattering cross sections of individual particle of any shape can be precisely determined. Outside these recognized strengths, numerical methods present also accepted weaknesses associated with their ease of implementation or required large computational memory and calculation time.

In complement to numerical methods, the present work illustrates the use of a simple group theory approach to address the plasmonics of subwavelength objects. The strengths of the method are 2-fold: (i) it gives access to the symmetry elements of the plasmonic eigenstates of an individual regular particle as a function of the symmetry of both the particle and the incident field. These charge eigenstates allow direct determination of the electric field distribution at the object surface given the polarization of the incident light. So, beyond its usefulness for field map interpretation, predictive manipulation of light through selective addressing of pure or combined charge eigenstates is also made possible, and (ii) it is an easy and quick analytic method. A full determination of charge eigenstates is obtained within a few-minutes handwork. Limitations are 
Table 1. Character Table for the $C_{4 v}$ Point Group

\begin{tabular}{lrrrrrll} 
& $E$ & $2 C_{4}(z)$ & $C_{2}$ & $2 \sigma_{v}$ & $2 \sigma_{d}$ & linear, rotations & quadratic \\
$A_{1}$ & 1 & 1 & 1 & 1 & 1 & $z$ & $x^{2}+y^{2}, z^{2}$ \\
$A_{2}$ & 1 & 1 & 1 & -1 & -1 & $R_{z}$ & \\
$B_{1}$ & 1 & -1 & 1 & 1 & -1 & & $x^{2}-y^{2}$ \\
$B_{2}$ & 1 & -1 & 1 & -1 & 1 & & $x y$ \\
$E$ & 2 & 0 & -2 & 0 & 0 & $(x, y)\left(R_{x}, R_{y}\right)$ & $(x z, y z)$ \\
\hline
\end{tabular}

mainly associated with the discrete character of the plasmonic charges and some arbitrariness in the choice of the initial basis set. Indeed, as mentioned in the following paragraphs, charges are rendered by s orbital like distributions attached to angular points of the object surface. In addition, no direct determinations of the LSP resonance absolute frequencies (at cross-section maxima) are possible.

In more detail, as recently demonstrated, the plasmonic response of a finite metallic structure can be predicted by analyzing the symmetry of both the object under investigation and the external incident field. Zhang et al. ${ }^{44}$ extended the selection rules established for a scalar field (a wave function) to the case of a vector field. This vectorial selection rule states that if the $i$ th component $(i=x, y, z)$ of the electric field $\vec{E}_{n}$ of a LSPR mode indexed $n$, respectively, of an external field, $\overrightarrow{E_{\text {ext }}}$, transforms as the irreducible representation (irrep) $\Gamma_{n, i}$, respectively, $\Gamma_{\text {ext }, i}$ then the excitation strength of the resonance mode $n\left\langle E_{n} \mid E_{\text {ext }}\right\rangle$ vanishes unless there is a product $\Gamma_{n, i} \otimes \Gamma_{\text {ext }, i}$ transforming as the totally symmetric irrep A.

In what follows, the case of a cubic particle illuminated by an incident plane wave is investigated, and to conform with experiment only the dipolar plasmon resonance $(l=1)$ is considered. A cube sitting on a substrate plane exhibits structural symmetries corresponding to the $C_{4 v}$ point group (see Supporting Information). Note that throughout the manuscript standard conventions are followed; in particular, the principal order rotation axis is taken as the normal $z$ axis of the Cartesian reference coordinate system $(\mathrm{O} x, \mathrm{O} y, \mathrm{O} z)$. According to the $C_{4 v}$ character table (Table 1), the irreps of the dipolar resonance modes are of symmetry $E$ and $A_{1}$. The 2dimensional (2D) irrep $E$ corresponds to an electric dipole within the $x y$ plane and the $1 \mathrm{D}$ irrep $A_{1}$ to a mode with its dipole aligned to the principal axis $z$ (normal to the substrate plane). On the other hand, the external field is vectorial in nature $\overrightarrow{E_{\mathrm{ext}}}=\left(E_{\mathrm{ext}, x} ; E_{\mathrm{ext}, y} ; E_{\mathrm{ext}, z}\right)$ and transforms like the translation vectors $(x, y, z)$. So, according to the stated selection rules, the $E$ or $A_{1}$ dipolar mode is optically active if excited by an electric field possessing a component, within the $x y$ plane or aligned along the $z$ axis, respectively (see Supporting Information for details). Both in-plane $E$ modes are degenerated in frequency; furthermore taking into account the regular dimensions of the cubic particle in all three directions, $\mathrm{E}$ and $\mathrm{A}_{1}$ modes should present close dipolar resonance frequencies and spectral overlap between LSPRs are to be expected.

Beyond pure symmetry considerations, group theory can also be used to obtain the charge distribution pattern associated with a specific resonance. Indeed, one LSP resonance corresponds to the accumulation of surface charges at specific angular points of the object: corners, edges, or faces. Either positive or negative, these induced charges can be described by virtual s-atomic orbitals attached to specific sites of the object surface. One plasmon resonance can thus be later on described within a linear combination of atomic orbitals approach (LCAO), and the subsequent determination of the symmetry adapted linear combinations (SALC) of orbitals does provide a direct insight regarding the charge distribution of the LSPR modes of the object under interest.

Figure 2 illustrates such an approach in the case of the dipolar resonance of a cube. As known from previous theoretical and experimental subwavelength imaging investigations, in a cube, the dipolar LSPR charges accumulate at the corner sites. ${ }^{17,21,22,45-51}$ We first consider the optically active dipolar LSPR of irreducible representation $E$. Its degeneracy is 2 -fold, meaning that the physical charge distribution of the resonance corresponds to linear combinations of a pair of twoeigenvector basis. The latter is obtained by application of the projector operator (van Vleck operator) to a basis function set. $^{52,53}$ As a result two orthogonal SALC polarization states are obtained. In more details, starting with a set of s-type orbitals mimicking either positive or negative charges at cube corners, the SALC eigenvector basis for the $E$ mode is constituted of the two charge combinations $(0,1,0,-1,0,1,0,-1)$ and $(1,0,-1$,

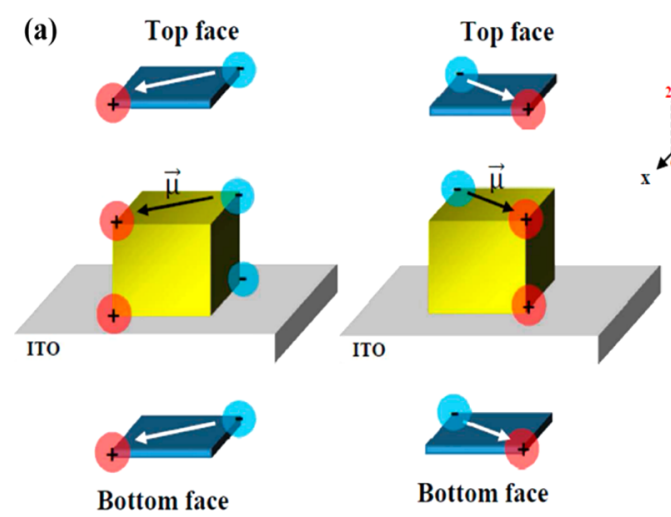

E symmetry

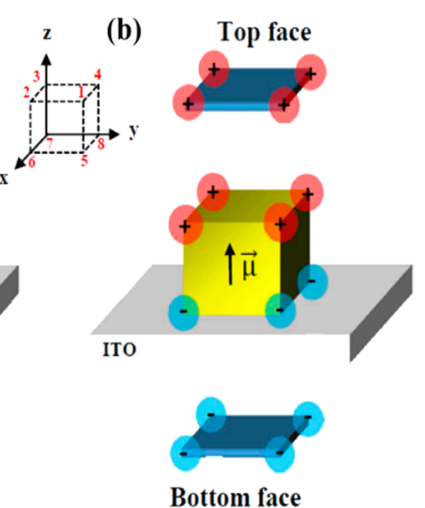

A symmetry

Figure 2. SALC eigenvector basis for the two $E$ and $A_{1}$ dipolar LSPR modes of a cube as determined by group theory for, respectively, in-plane $\overrightarrow{E_{\text {ext }}}$ $=\left(E_{\text {ext }, x} ; E_{\text {ext }, y} ; 0\right)$ (left) and out-of-plane $\overrightarrow{E_{\text {ext }}}\left(0 ; 0 ; E_{\text {ext }, z}\right)$ (right) incident light. For simplicity, top and bottom charges are represented by similar sphere symbols. 


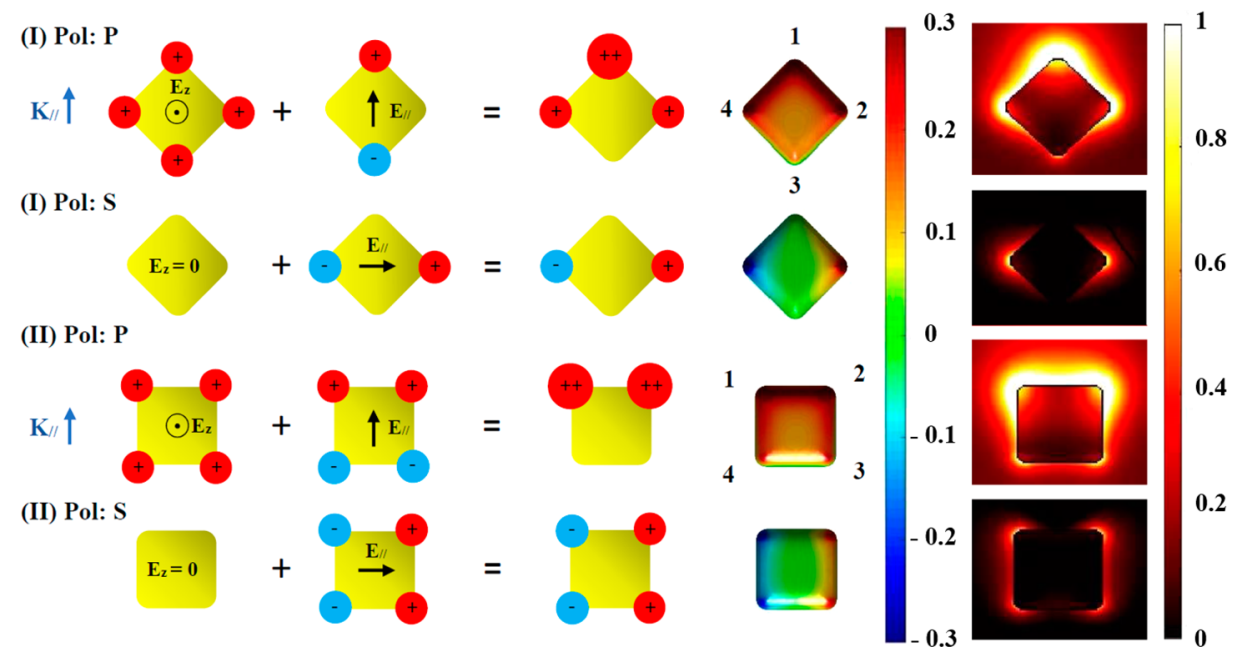

Figure 3. Dipolar resonances of a cube for simple illuminating geometries, grazing incidence $72.5^{\circ}$ off normal axis, and p- and s-polarizations. Left: charge distributions as predicted by group theory. Middle and right columns: BEM numerical simulations, charge distributions, and $I E_{\text {tot }} \mid$ field maps at the top cube face. Cube edge dimensions $60 \mathrm{~nm}(<165 \mathrm{~nm}$ to minimize scattering contribution). Linear color scale.

$0,1,0,-1,0)$, where the number sequences represent the top and bottom corner positions (Figure 2, inset). These orthogonal eigenvectors correspond to the diagonals of the top and bottom faces (see Figure 2a). For the $A_{1}$ dipolar mode, the same route can be followed. This time its degeneracy is of value 1 , and the dipolar $A_{1}$ resonance corresponds to a unique eigenstate vector described in Figure $2 \mathrm{~b}$. Its polarization is aligned along the $z$ vertical axis and can be described by the charged corner sequence $(1,1,1,1,-1,-1,-1,-1)$. All these resonance states can be selectively excited via a proper choice of the polarization of the incident light field. Note that the $C_{4 v}$ point group does not contain any inversion symmetry with respect to the substrate plane (normal to $z$ axis), so top and bottom charges are to be expected of different values as found in digital simulations. ${ }^{47}$

Photoemission experiments on nanocubes can be easily interpreted as combinations of the $E$ and $A_{1}$ LSPR resonances as displayed in Figure 3. Indeed, any incident electrical field can be decomposed onto the SALC eigenstate vector basis. In the case of a dipolar resonance, the in-plane components determine the relative weights of the modes of $E$ symmetry; equivalently the $z$ component determines the $A_{1}$ mode contribution. Linear combinations of the weighed SALC contributions then yield the expected total charge distributions at resonance. As stated above, linear combinations of $E$ and $A_{1}$ modes are made possible thanks to the closeness of their respective resonance wavelengths (spectral overlap). Figure 3 sums up the predicted charge distributions for simple geometrical configurations of interest for the case of a dipolar resonance. Under grazing incidence and for electric field in p-polarization, two limiting cases can be predicted depending on the relative direction of the $\mathbf{k}_{\text {// }}$ wavevector. Case I, with the $\mathbf{k}_{/ /}$in-plane vector aligned on the cube diagonal corresponds to the simultaneous excitations of two SALC eigenvectors, namely, one of the inplane $E$ modes and the vertical $A_{1}$ mode. The obtained charge distribution exhibits surface charges at corner positions according to the sequence $(+2,+1,0,+1,0,-1,-2,-1)$. Considering only the top corners, it corresponds to the transverse diagonal ones (corners $\left.n^{\circ} 2,4\right)$ and the ones aligned along the beam direction and situated at the opposite edge with respect to the incident illuminating light (corner $\left.n^{\circ} 1\right)$. The corner at the leading position (corner 3) is systematically extinguished (reversed case for the bottom positions). In case II, the in-plane beam wavevector $\mathbf{k}_{/ /}$is perpendicular to two of the cube faces, and charge summation then yields a charged corner sequence $(+2,+2,0,0,0,0,-2,-2)$ corresponding to a systematic extinction of the two top leading corners (corners $n^{\circ}$ $3,4)$ and strong signals at the opposite ones (corners $n^{\circ} 1,2$ ); the bottom cube face adopts a reversed charge distribution. Note that the systematic extinctions predicted by the group theory approach do correspond to the retardation field effects encountered in previous investigations. ${ }^{17}$ The charge schemes predicted for s-polarization are reported in Figure 3, lines 2 and 4.

\section{BOUNDARY ELEMENT METHOD}

The group theory analysis is complemented by numerical simulations based on the boundary element method (BEM) via its implementation in the multiple nanoparticles BEM MNPBEM toolbox. ${ }^{54,55}$ The BEM approach provides solutions of Maxwell's equations in the case of arbitrarily shaped abrupt dielectric interfaces. The solutions are expressed in terms of surface charges and currents, so that only surfaces between the different materials have to be discretized, providing fast simulations. The BEM method is related to the surface integral equation method. ${ }^{43}$ For more details, see refs $18-20$.

The Au nanocubes are modeled on an infinite ITO layer whose refractive index is fixed at $n_{\text {ITO }}=2$. The refractive index of $\mathrm{Au}$ is taken from Johnson and Christy. ${ }^{56}$ Simulations are carried out in a geometry corresponding to the one used in the PEEM experiment, namely, a light beam illuminating the sample at a grazing incidence of $72.5^{\circ}$ off the normal axis. In a photoemission experiment, the electron yield scales as an even power of the surface electric field, more precisely its normal component to the interface taken on the metal side. ${ }^{27,57-59}$ The nonlinearity order $n$ corresponds to the minimum number of photon absorption events necessary for one electron to overcome the material work function. For wavelength in the visible range below $545 \mathrm{~nm}$, the experimentally determined nonlinearity order for $\mathrm{Au}$ (work function $4.5-4.7 \mathrm{eV}^{60}$ ) is 2 . Further on, in connection to the limited acceptance angle of the PEEM imaging column, electrons photoemitted from lateral 

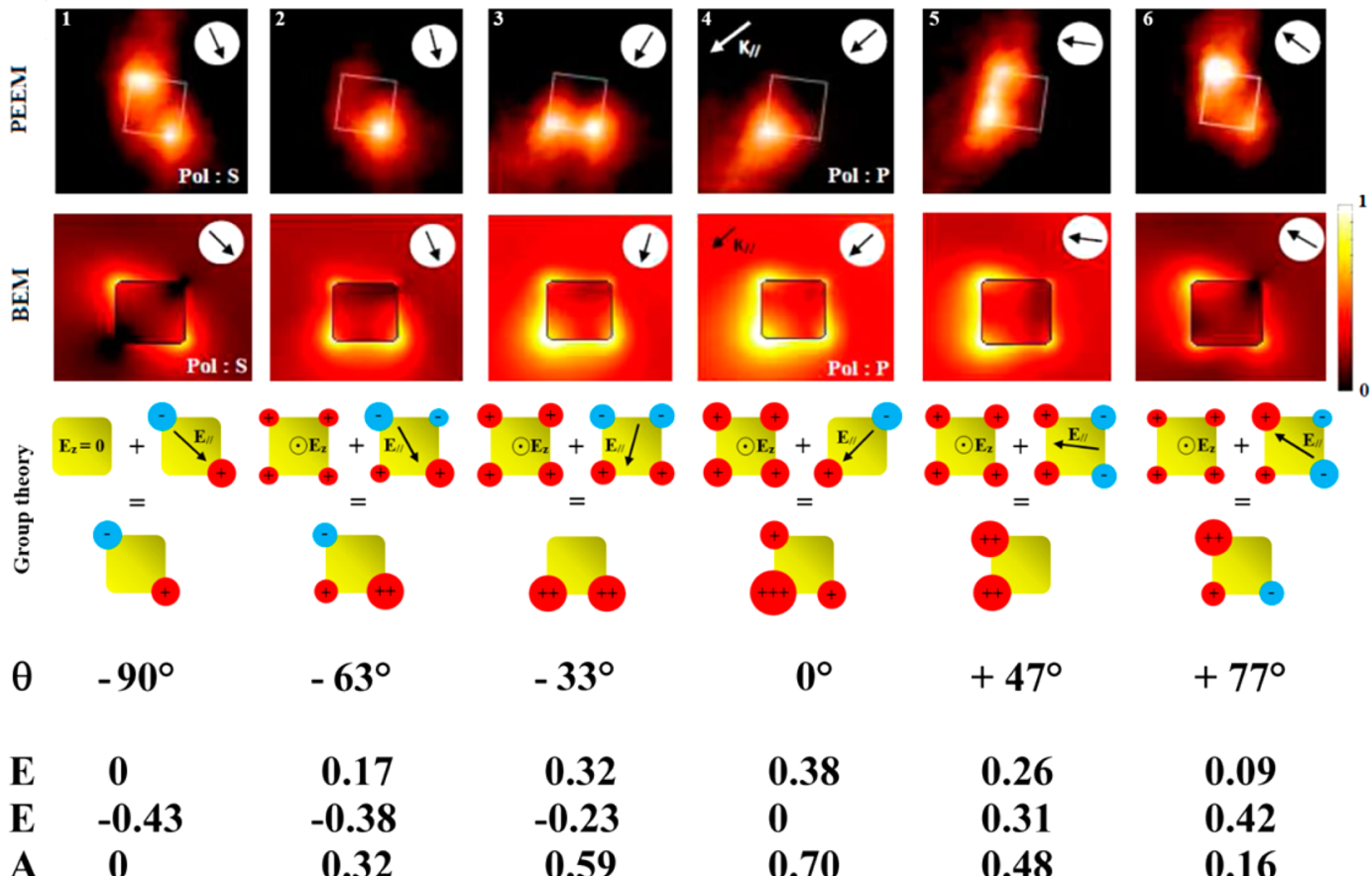

$-33^{\circ}$

$0^{\circ}$

$+47^{\circ}$

$+77^{\circ}$

$\begin{array}{llrr}\text { E } & 0 & 0.17 & 0.32 \\ \text { E } & -\mathbf{0 . 4 3} & -\mathbf{0 . 3 8} & -\mathbf{0 . 2 3} \\ \text { A } & 0 & \mathbf{0 . 3 2} & \mathbf{0 . 5 9}\end{array}$

0.38
0
0.70

0.26
0.31
0.48

0.09

0.42

0.16

Figure 4. Dipolar resonances of a cube as investigated by PEEM. Cube edge dimension $165 \mathrm{~nm}$. Green visible light, wavelength $541 \mathrm{~nm}$. Illumination at grazing incidence $72.5^{\circ}$ off normal axis. Top sequence: 2-hv PEEM signature under $\theta$ polarization angles $\left\{-90^{\circ}(\mathrm{S}),-63^{\circ},-33^{\circ}, 0^{\circ}(\mathrm{P}),+47^{\circ}\right.$, $\left.+77^{\circ}\right\}$. Middle sequence: BEM numerical simulations, $\left|E_{\text {tot }}\right|^{4}$ field maps, log color scale. Bottom sequence: group theory charge predictions. $\theta$ polarization angles, exciting field components along the directions of the resonance eigenstates $\left(E, E, A_{1}\right)=([110]$, $[-110]$, [001]), reported inplane exciting field take into account Fresnel coefficients (Supporting Information).

faces do not contribute significantly to the final photoelectric signal. So, in the numerical simulations only photoelectrons emitted from the top face of the cube particles are taken into account, and the PEEM electron maps are calculated as the time-averaged version of the quantity $\int_{\text {Top face }}\left(\vec{E}_{\text {int. }, z}(\vec{r})\right)^{4} \mathrm{~d} r$, where $\vec{E}_{\text {int. }, z}(\vec{r})$ designs the vertical $z$ component of the internal electric field at the object surface (metal side). The wavelength used in the simulations corresponds to the wavelength at the calculated absorption cross-section maximum. These simulations allowed us to map the electric field at the vicinity of the particle surface and to calculate the absorption and scattering spectra of the particle under interest.

Simulated charge distributions and near-field maps for the illumination geometries corresponding to the ones considered above are displayed in Figure 3, in the two right columns. Whatever the respective orientations of the light beam wavevector and electrical field polarization, simulated charge and $|E|$ field maps do reproduce the predicted surface charge distributions obtained within the group theory approach. Beyond symmetry considerations, a qualitative agreement is also visible; i.e., relative surface charges do qualitatively scale with simulated nonlinear field intensities.

\section{RESULTS AND DISCUSSION}

Figure 4 sums up a representative sequence of the experimental photoemission signatures of one peculiar cube particle upon polarization change under grazing incidence. The exciting wavelength of $541 \mathrm{~nm}$ is close to its dipolar resonance wavelength (green light). Note that cubes are randomly oriented at the surface, and the one retained object does simply offer a geometry of interest with the in-plane wavevector component $\mathbf{k}_{/ /}$aligned along its diagonal. For completeness, simulated BEM $\left|E_{\text {tot }}\right|^{4}$ field maps taking into consideration the exact illumination geometries are also presented. Additional sequences acquired on other cube particles can be found in the Supporting Information.

Image 4.1 figures out the response of the cube when illuminated in s-polarization. Alignment of the in-plane polarization along one of the cube diagonals allows for the direct excitation of one well-defined $E$ symmetry eigenstate. Correspondingly, the two aligned and diagonally opposed corners are fully excited.

At polarization angle $\theta=-33^{\circ}$ and more specifically $+47^{\circ}$ polarization angles (Figure 4.3 and 4.5 ), the in-plane exciting field orients itself gradually toward the face normal direction. Accordingly the two opposite face corners light up due to simultaneous excitations of all three resonance eigenstates $(E$, $\left.E, A_{1}\right)$.

Polarization angles $\theta=\left(-63^{\circ},+77^{\circ}\right)$ (Figure 4.2 and 4.6) correspond to intermediate exciting geometries between the spolarization and the normal to face cases. Three of the four cube top corners exhibit a significant photoemission signal, in full agreement with BEM simulation and the group theory.

Further rotation of the polarization angle yields illumination in the p-polarization state (Figure 4.4. Visual inspection shows one strong unique near-field hot spot at the corner opposite the incident light beam in apparent contradiction with both group theory prediction and BEM simulation where three spots are expected. As a matter of fact, the missing of the two transverse spots in the experimental picture comes from an insufficient dynamics of the PEEM detection line. Indeed, being nonlinear in nature, the photoemission signal exhibits a large dynamics challenging the detector capabilities. Slight off detuning from the p-polarization, case $\theta=-33^{\circ}$ (Figure 4.3), allows for a more favorable balance of the relative hot spot intensities and 
full agreement between experiment and BEM simulation, and group theory prediction is recovered.

\section{CONCLUSIONS}

In conclusion, a simple group theory approach is proposed to interpret and predict the low-order localized surface plasmon resonances of finite particles of basic geometries. As an illustration, the case of randomly oriented Au colloidal particles of cubic $C_{4 v}$ symmetry excited at dipolar resonance is presented. The symmetry approach is confirmed by numerical simulations carried out by the boundary element method. Experimental validation is achieved by high-resolution (subwavelength) nearfield mapping conducted by two-photon photoemission electron microscopy. This investigation confirms the general character of the group theory approach previously applied to the specific case of planar objects of trigonal symmetry. ${ }^{15}$ On a wider perspective, this work opens the way to a simple light manipulation at a subwavelength scale by direct and easy polarization addressing of one or combined particle resonance eigenstates.

\section{ASSOCIATED CONTENT}

\section{S Supporting Information}

The Supporting Information is available free of charge on the ACS Publications website at DOI: 10.1021/acs.jpcc.6b10585.

SEM images of prepared cube particles. Selection rules for a $C_{4 v}$ cube particle excited by a plane-wave, analytical exciting electrical field, and polarization dependence are detailed. Additional sequences acquired on cube particles of various orientations are presented. In addition, the predicted LSPR charge eigenstates of regular particles closely related to the cubic case are presented, namely, the square and rectangular plate cases (PDF)

\section{AUTHOR INFORMATION}

\section{Corresponding Author}

*E-mail: ludovic.douillard@cea.fr.

\section{ORCID ${ }^{\circ}$}

Fabrice Charra: 0000-0003-1228-0583

Ludovic Douillard: 0000-0001-6370-6586

\section{Notes}

The authors declare no competing financial interest.

\section{ACKNOWLEDGMENTS}

The CEA authors acknowledge financial support by the French National Agency (ANR) in the frame of its program in Nanosciences and Nanotechnologies (PEEMPlasmon Project ANR-08-NANO-034), Nanosciences Île-de-France (PEEMPlasmonics project), and the "Triangle de la Physique" (PEPS Project 2012-035T). Support of S. Vassant in the figure preparation was greatly appreciated.

\section{REFERENCES}

(1) Mie, G. Beiträge zur Optik trüber Medien, speziell kolloidaler Metallösungen. Ann. Phys. (Berlin, Ger.) 1908, 330, 377-445.

(2) Zhong, Y.; Ueno, K.; Mori, Y.; Shi, X.; Oshikiri, T.; Murakoshi, $\mathrm{K}$; Inoue, H.; Misawa, H. Plasmon-assisted water splitting using two sides of the same $\mathrm{SrTiO}_{3}$ single-crystal substrate: conversion of visible light to chemical energy. Angew. Chem., Int. Ed. 2014, 53, 1035010354.
(3) Oshikiri, T.; Ueno, K.; Misawa, H. Plasmon-induced ammonia synthesis through Nitrogen photofixation with visible light irradiation. Angew. Chem., Int. Ed. 2014, 53, 9802-9805.

(4) Yanik, A. A.; Cetin, A. E.; Huang, M.; Artar, A.; Mousavi, S. H.; Khanikaev, A.; Connor, J. H.; Shvets, G.; Altug, H. Seeing protein monolayers with naked eye through plasmonic Fano resonances. Proc. Natl. Acad. Sci. U. S. A. 2011, 108, 11784-11789.

(5) Yanik, A. A.; Huang, M.; Kamohara, O.; Artar, A.; Geisbert, T. W.; Connor, J. H.; Altug, H. An Optofluidic nanoplasmonic biosensor for direct detection of live viruses from biological media. Nano Lett. 2010, 10, 4962-4969.

(6) Jin, Y. Engineering Plasmonic gold nanostructures and metamaterials for biosensing and nanomedicine. Adv. Mater. 2012, $24,5153-5165$

(7) Huang, X.; Jain, P. K.; El-Sayed, I. H.; El-Sayed, M. A. Plasmonic photothermal therapy (PPTT) using gold nanoparticles. Lasers Med. Sci. 2008, 23, 217-228.

(8) Atwater, H. A.; Polman, A. Plasmonics for improved photovoltaic devices. Nat. Mater. 2010, 9, 205-213.

(9) Kawata, S.; Inouye, Y.; Verma, P. Plasmonics for near-field nanoimaging and superlensing. Nat. Photonics 2009, 3, 388-394.

(10) Yang, A.; Hoang, T. B.; Dridi, M.; Deeb, C.; Mikkelsen, M. H.; Schatz, G. C.; Odom, T. W. Real-time tunable lasing from plasmonic nanocavity arrays. Nat. Commun. 2015, 6, 6939.

(11) Hatab, N. A.; Hsueh, C.-H.; Gaddis, A. L.; Retterer, S. T.; Li, J.H.; Eres, G.; Zhang, Z.; Gu, B. Real-time tunable lasing from plasmonic nanocavity arrays. Nano Lett. 2010, 10, 4952-4955.

(12) Renishaw Apply Innovation (C) http://www.renishaw.com (accessed January 3, 2017).

(13) Aeschlimann, M.; Bauer, M.; Bayer, D.; Brixner, T.; García de Abajo, F. J.; Pfeiffer, W.; Rohmer, M.; Spindler, C.; Steeb, F. Adaptive subwavelength control of nano-optical fields. Nature 2007, 446, 301.

(14) Melchior, P.; Bayer, D.; Schneider, C.; Fisher, A.; Rohmer, M.; Pfeiffer, W.; Aeschlimann, M. Optical near-field interference in the excitation of a bowtie nanoantennas. Phys. Rev. B: Condens. Matter Mater. Phys. 2011, 83, 235407.

(15) Awada, C.; Popescu, T.; Douillard, L.; Charra, F.; Perron, A.; Yockell-Lelièvre, H.; Baudrion, A.-L.; Adam, P.-M.; Bachelot, R Selective excitation of plasmon resonances of single Au triangles by polarization-dependent light excitation. J. Phys. Chem. C 2012, 116, 14591-14598.

(16) Hrelescu, C.; Sau, T. K.; Rogach, A. L.; Jäckel, F.; Laurent, G.; Douillard, L.; Charra, F. Selective excitation of individual plasmonic hotspots at the tips of single gold nanostars. Nano Lett. 2011, 11, 402407.

(17) Mårsell, E.; Svärd, R.; Miranda, M.; Guo, C.; Harth, A.; Lorek, E.; Mauritsson, J.; Arnold, C. L.; Xu, H.; L'Huillier, A.; et al. Direct subwavelength imaging and control of near-field localization in individual silver nanocubes. Appl. Phys. Lett. 2015, 107, 201111201111-5.

(18) Garcia de Abajo, F. J.; Howie, A. Retarded field calculation of electron energy loss in inhomogeneous dielectrics. Phys. Rev. B: Condens. Matter Mater. Phys. 2002, 65, 115418.

(19) Garcia de Abajo, F. J. Optical excitations in electron microscopy. Rev. Mod. Phys. 2010, 82, 209.

(20) Hohenester, U.; Trügler, A. Interaction of single molecules with metallic nanoparticles. IEEE J. Sel. Top. Quantum Electron. 2008, 14, 1430.

(21) Haggui, M.; Dridi, M.; Plain, J.; Marguet, S.; Perez, H.; Schatz, G. C.; Wiederrecht, G. P.; Gray, S. K.; Bachelot, R. Spatial confinement of electromagnetic hot and cold spots in gold nanocubes. ACS Nano 2012, 6, 1299-1307.

(22) Deeb, C.; Zhou, X.; Miller, R.; Gray, S. K.; Marguet, S.; Plain, J.; Wiederrecht, G. P.; Bachelot, R. Mapping the electromagnetic nearfield enhancements of gold nanocubes. J. Phys. Chem. C 2012, 116, 24734-24740.

(23) Yang, J.; Liu, H.; Martens, W. N.; Frost, R. L. Synthesis and characterization of cobalt hydroxide, cobalt oxyhydroxide, and cobalt oxide nanodiscs. J. Phys. Chem. C 2010, 114, 111-119. 
(24) Eguchi, M.; Mitsui, D.; Wu, H.-L.; Sato, R.; Teranishi, T. Simple reductant concentration-dependent shape control of polyhedral gold nanoparticles and their plasmonic properties. Langmuir 2012, 28, 9021-9026.

(25) Präzisions Glas \& Optik GmbH, CEC050P, ITO-coating ( $\leq 50$ Ohms/sq. on polished glass substrate $\mathrm{SiO}_{2}$ ).

(26) Douillard, L.; Charra, F.; Fiorini, C.; Adam, P.-M.; Bachelot, R.; Kostcheev, S.; Lerondel, G.; Lamy de la Chapelle, M.; Royer, P. Optical properties of metal nanoparticles as probed by photoemission electron microscopy. J. Appl. Phys. 2007, 101, 083518.

(27) Douillard, L.; Charra, F. High-resolution mapping of plasmonic modes: photoemission and scanning tunnelling luminescence microscopies. J. Phys. D: Appl. Phys. 2011, 44, 464002.

(28) Schmidt, O.; Bauer, M.; Wiemann, C.; Porath, R.; Scharte, M.; Andreyev, O.; Schonhense, G.; Aeschlimann, M. Time-resolved two photon photoemission electron microscopy. Appl. Phys. B: Lasers Opt. 2002, 74, 223-227.

(29) Meyer zu Heringdorf, F.-J.; Chelaru, L. I.; Möllenbeck, S.; Thien, D.; Horn-von Hoegen, M. Femtosecond photoemission microscopy. Surf. Sci. 2007, 601, 4700-4705.

(30) Buckanie, N. M.; Kirschbaum, P.; Sindermann, S.; Meyer zu Heringdorf, F.-J. Interaction of light and surface plasmon polaritons in $\mathrm{Ag}$ Islands studied by nonlinear photoemission microscopy. Ultramicroscopy 2013, 130, 49-53.

(31) Kubo, A.; Onda, K.; Petek, H.; Sun, Z. J.; Jung, Y. S.; Kim, H. K. Femtosecond imaging of surface plasmon dynamics in a nanostructured silver film. Nano Lett. 2005, 5, 1123-1127.

(32) Messinger, B. J.; von Raben, K. U.; Chang, R. K.; Barber, P. W. Local fields at the surface of noble-metal microspheres. Phys. Rev. B: Condens. Matter Mater. Phys. 1981, 24, 649-657.

(33) Ross, B. M.; Lee, L. P. Comparison of near- and far-field measures for plasmon resonance of metallic nanoparticles. Opt. Lett. 2009, 34, 896-898.

(34) Yorulmaz, M.; Nizzero, S.; Hoggard, A.; Wang, L.-Y.; Cai, Y.-Y.; Su, M.-N.; Chang, W.-S.; Link, S. Single-particle absorption spectroscopy by photothermal contrast. Nano Lett. 2015, 15, 3041-3047.

(35) Yee, K. Numerical solution of initial boundary value problems involving Maxwell's equations in isotropic media. IEEE Trans. Antennas Propag. 1966, 14, 302.

(36) Taflove, A.; Hagness, S. C. Computational Electrodynamics: The Finite-Difference Time-Domain Method; Artech House: Boston, MA, 2005.

(37) Montgomery, J. M.; Lee, T. W.; Gray, S. K. Theory and modeling of light interactions with metallic nanostructures. J. Phys.: Condens. Matter 2008, 20, 323201.

(38) Jin, J.-M. The Finite Element Method in Electromagnetics, 3rd ed.; Wiley-IEEE Press, 2014.

(39) Martin, O. J. F.; Piller, N. B. Electromagnetic scattering in polarizable backgrounds. Phys. Rev. E: Stat. Phys., Plasmas, Fluids, Relat. Interdiscip. Top. 1998, 58, 3909-3915.

(40) Draine, B. T.; Flatau, P. J. Discrete-dipole approximation for scattering calculations. J. Opt. Soc. Am. A 1994, 11, 1491-1499.

(41) Kern, A. M.; Martin, O. J. F. Surface integral formulation for 3D simulations of plasmonic and high permittivity nanostructures. J. Opt. Soc. Am. A 2009, 26, 732-740.

(42) Taboada, J. M.; Rivero, J.; Obelleiro, F.; Araujo, M. G.; Landesa, L. Method-of-moments formulation for the analysis of plasmonic nano-optical antennas. J. Opt. Soc. Am. A 2011, 28, 1341-1348.

(43) Gallinet, B.; Butet, J.; Martin, O. J. F. Numerical methods for nanophotonics: standard problems and future challenges. Las. Photo. Rev. 2015, 9, 577-603.

(44) Zhang, W.; Gallinet, B.; Martin, O. J. F. Symmetry and selection rules for localized surface plasmon resonances in nanostructures. Phys. Rev. B: Condens. Matter Mater. Phys. 2010, 81, 233407.

(45) Zhou, F.; Li, Z.-Y.; Liu, Y.; Xia, Y. Quantitative analysis of dipole and quadrupole excitation in the surface plasmon resonance of metal nanoparticles. J. Phys. Chem. C 2008, 112, 20233-20240.
(46) Kim, D.-S.; Heo, J.; Ahn, S.-H.; Han, S. W.; Yun, W. S.; Kim, Z. $\mathrm{H}$. Real-space mapping of the strongly coupled plasmons of nanoparticle dimers. Nano Lett. 2009, 9, 3619-3625.

(47) Ringe, E.; McMahon, J. M.; Sohn, K.; Cobley, C.; Xia, Y.; Huang, J.; Schatz, G. C.; Marks, L. D.; Van Duyne, R. P. Unraveling the effects of size, composition, and substrate on the localized surface plasmon resonance frequencies of gold and silver nanocubes: a systematic single-particle approach. J. Phys. Chem. C 2010, 114, 12511-12516.

(48) Cortie, M. B.; Liu, F.; Arnold, M. D.; Niidome, Y. Multimode resonances in silver nanocuboids. Langmuir 2012, 28, 9103-9112.

(49) Mazzucco, S.; Geuquet, N.; Ye, J.; Stéphan, S.; Van Roy, W.; Van Dorpe, P.; Henrard, L.; Kociak, M. Ultralocal modification of surface plasmons properties in silver nanocubes. Nano Lett. 2012, 12, $1288-1294$.

(50) Nicoletti, O.; de la Peña, F.; Leary, R. K.; Holland, D. J.; Ducati, C.; Midgley, P. A. Three-dimensional imaging of localized surface plasmon resonances of metal nanoparticles. Nature 2013, 502, 80.

(51) Sun, Q.; Yu, H.; Ueno, K.; Kubo, A.; Matsuo, Y.; Misawa, H. Dissecting the few-femtosecond dephasing time of dipole and quadrupole modes in gold nanoparticles using polarized photoemission electron microscopy. ACS Nano 2016, 10, 3835-3842.

(52) Walton, P. H. Chimie Et Théorie Des Groupes; De Boeck Edt, 2001.

(53) Bradley, C.; Cracknell, A. The Mathematical Theory of Symmetry in Solids: Representation Theory for Point Groups and Space Groups; Oxford Classic Texts in the Physical Sciences, OUP: Oxford, 2009.

(54) Hohenester, U.; Trügler, A. MNPBEM - A Matlab toolbox for the simulation of plasmonic nanoparticles. Comput. Phys. Commun. 2012, 183, 370-381.

(55) Waxenegger, J.; Trügler, A.; Hohenester, U. Plasmonics simulations with the MNPBEM toolbox: consideration of substrates and layer structures. Comput. Phys. Commun. 2015, 193, 138-150.

(56) Johnson, P. B.; Christy, R. W. Optical constants of the noble metals. Phys. Rev. B 1972, 6, 4370-4379.

(57) Georges, A. T. Theory of the multiphoton photoelectric effect: A stepwise excitation process. Phys. Rev. B: Condens. Matter Mater. Phys. 1995, 51, 13735.

(58) Georges, A. T. High-order multiphoton photoelectric effect at midinfrared laser wavelengths. Phys. Rev. A: At., Mol., Opt. Phys. 2002, 66, 063412.

(59) Ferrini, G.; Banfi, F.; Giannetti, C.; Parmigiani, F. Non-linear electron photoemission from metals with ultrashort pulses. Nucl. Instrum. Methods Phys. Res., Sect. A 2009, 601, 123-131.

(60) Kawano, H. Effective work functions for ionic and electronic emissions from mono- and polycrystalline surfaces. Prog. Surf. Sci. 2008, 83, 1-165. 